\title{
UAS SATELLITE EARTH STATION EMISSION LIMITS FOR TERRESTRIAL SYSTEM INTERFERENCE PROTECTION
}

\author{
Robert J. Kerczewski, NASA Glenn Research Center, Cleveland, Ohio, USA, \\ and William D. Bishop Jacobs Engineering, NASA UAS Support, Cleveland, OH, USA
}

\begin{abstract}
Unmanned aircraft systems (UAS) will have a major impact on future aviation. Medium and large UA operating at altitudes above 3000 feet will require access to non-segregated, that is, controlled airspace. In order for unmanned aircraft to be integrated into the airspace and operate with other commercial aircraft, a very reliable command and control (C2, a. k. a. control and non-payload communications, (CNPC)) link is required. For operations covering large distances or over remote locations, a beyond-line-of-sight (BLOS) CNPC link would need to be implemented through satellite.
\end{abstract}

Significant progress has taken place on several fronts to advance the integration of UAS into controlled airspace, including the recent completion of Minimum Operational Performance Standards (MOPS) for terrestrial line-of-sight (LOS) UAS command and control (C2) links. The development of MOPS for beyond line-of-sight $\mathrm{C} 2$ satellite communication links is underway. Meanwhile the allocation of spectrum for UAS $\mathrm{C} 2$ by the International Telecommunications Union Radiocommunication Sector (ITU-R) has also progressed. Spectrum for LOS C2 was allocated at the 2012 World Radiocommunication Conference (WRC-12), and for BLOS C2 an allocation was made at WRC-15, under WRC-15 Resolution 155. Resolution 155, however, does not come into effect until several other actions have been completed. One of these required actions is the identification of a power flux density (pfd) limit on the emissions of UAS Ku-Band satellite communications transmitters reaching the ground. The pfd limit is intended to protect terrestrial systems from harmful interference. WRC-19 is expected to finalize the pfd limit. In preparation for WRC-19, analyses of the required pfd limit are on-going, and supporting activities such as propagation modeling are also planned. This paper provides the status of these activities.

\section{Introduction}

Progress on the development and implementation of UAS continues at a rapid pace. Ownership of small UAS operating at low (below $500 \mathrm{ft}$ ) altitudes in uncontrolled airspace has already reached millions of units, with personal and commercial applications too numerous to mention. Medium and large UAS will operate above $500 \mathrm{ft}$ and require at least occasional access to controlled airspace. In order for unmanned aircraft to be integrated into the airspace and operate with other commercial aircraft, a very reliable command and control (a. k. a. control and non-payload communications, (CNPC)) link is required. For operations covering large distances or over remote locations, a beyond-line-of-sight (BLOS) CNPC link implemented through a satellite is required. Protected aviation spectrum (aeronautical mobile satellite (route) service, or $\mathrm{AMS}(\mathrm{R}) \mathrm{S}$ ) would normally be used for such a safety-critical link, however studies have shown that currently available aviation safety satellite spectrum is inadequate to support the projected BLOS C2 link bandwidth requirements.

To address this inadequacy, the 2015 World Radiocommunication Conference (WRC-15) provided a provisional allocation allowing the use of the Fixed Satellite Service (FSS) to provide UAS BLOS C2, including allocations in $\mathrm{Ku}-\mathrm{B}$ and and $\mathrm{Ka}-$ Band, under WRC-15 Resolution 155. Although it was shown that UA CNPC satellite links in these bands can meet operational availability and continuity requirements, a serious complication exists in that there are also terrestrial service allocations in these bands, in particular, Fixed Service (FS) pointto-point and point-to-multipoint microwave digital links. During the WRC-15 study cycle, much opposition to AI 1.5 was generated based on fears that UA C2 satellite transmitters in these bands would impose unacceptable levels of interference to the FS receivers. 
Resolution 155 provided an allocation in KaBand that does not have a co-primary FS allocation, so the need to address protection of the FS receivers is limited to the Ku-Band uplink band allocation of 14.0-14.47 GHz. Resolution 155 contains a requirement to develop a power flux density (pfd) limit to be imposed on UA transmitters sufficient to protect FS receivers from harmful interference. The Resolution left the details of the pfd limit undefined, to be agreed upon at the next WRC in 2019. Thus, work is continuing to prepare technical arguments regarding selection of a pfd limit adequate to protect FS receivers in preparation for WRC-19.

New studies are now underway proposing appropriate pfd limits to be approved at WRC-19. In addition, NASA Glenn Research Center (GRC) will study propagation aspects of the interference path through measurements and modeling. The following sections provide an overview of the interference issue, an update on current proposals for pfd limits, and planned propagation measurement and interference channel modeling.

\section{Interference Environment}

Figure 1 describes the operational scenario for BLOS C2 satellite communications links operating on a co-primary basis with FS stations. The link labelled $3 \mathrm{~s}$ is the interference path from the UAS satellite transmitter into the terrestrial FS receiver. Several iterations of sharing studies performed for WRC-15 indicated varying impacts from the UAS on the FS receiver depending upon operational parameters of the UAS [1-3].

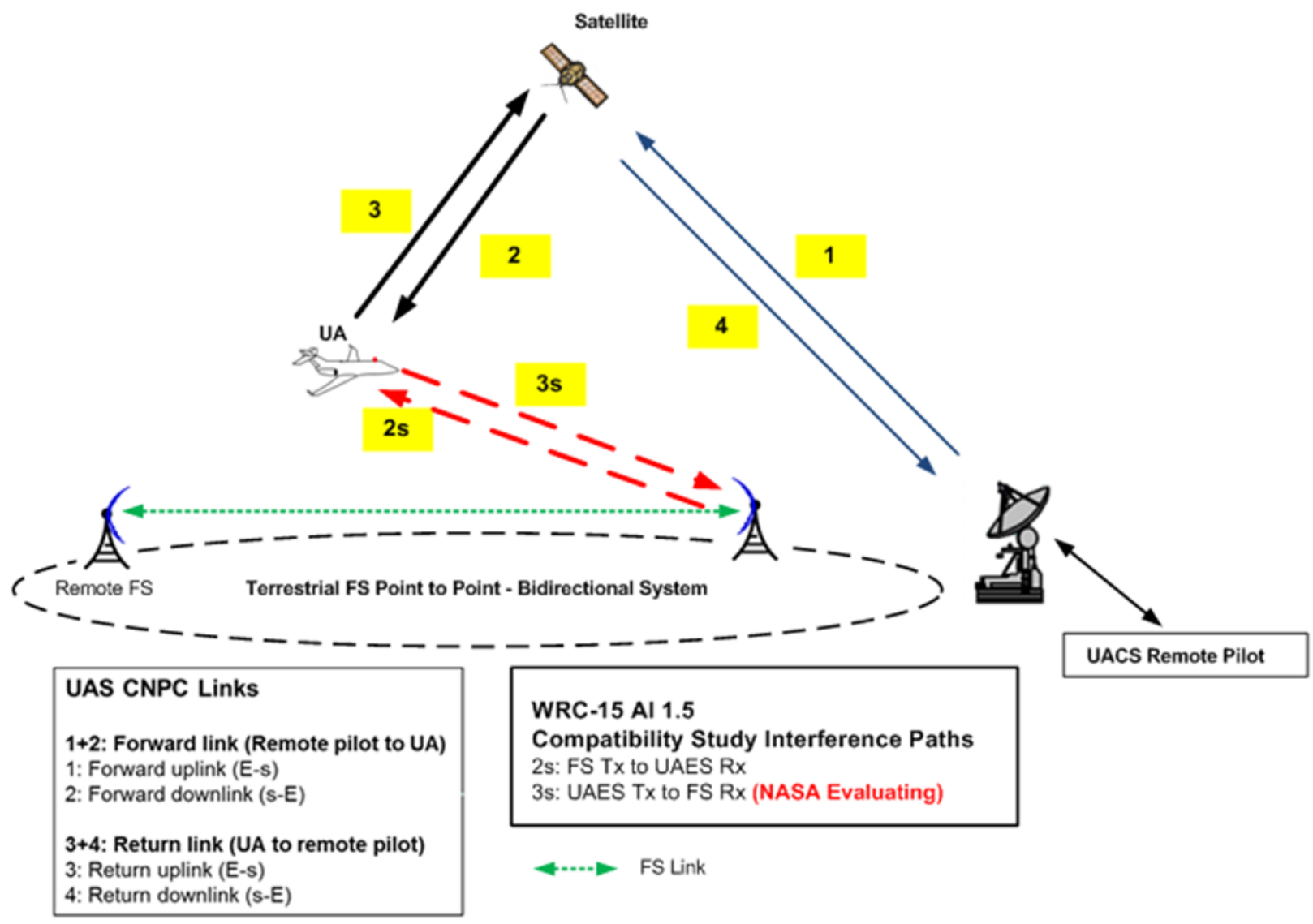

Figure 1 - UA Satellite Communications C2 BLOS Links under Study 
The protection required for the FS receiver is expressed as the ratio of interference power to noise power, I/N. Interference protection is considered for both long term and short term time intervals. Higher interference levels are defined for very brief time periods, while lower interference levels can persist for longer periods of time. Table 1 shows the allowable interference levels at $14.4 \mathrm{GHz}$ [4].

Table 1 - Interference protection criteria for the Fixed Service at 14.4 GHz

\begin{tabular}{|c|l|l|}
\hline \multicolumn{1}{|c|}{ Parameter } & Value & \multicolumn{1}{|c|}{ Comments } \\
\hline Long-Term I/N (dB) & $-10 \mathrm{~dB}$ & $\begin{array}{l}\text { Not to exceed for } \\
\text { more than } 20 \% \text { of the } \\
\text { time }\end{array}$ \\
\hline Short-Term I/N (dB) & $+20 \mathrm{~dB}$ & $\begin{array}{l}\text { Not to exceed for } \\
\text { more than } 1 \times 10^{-4} \% \\
\text { of the time. }\end{array}$ \\
\hline
\end{tabular}

\section{Figure 3. C2 Radio Flight Test Tracks for Several Flights in Northern and Southern Ohio}

In analyzing interference between the UAS and FS, the key parameters include the UAS satellite antenna and FS receiver antenna patterns, UAS operating altitude, latitude of the FS station, UAS transmitted power, FS antenna elevation angle, satellite longitude relative to UAS position, and the density of UAS operating within view of the FS station.

Previous sharing performed by NASA indicated that in most situations the UAS can operate without causing harmful interference $[1,3]$. The impact of the key parameters was analyzed [2], and studies made use of the worst case for several key parameters. Other parameters are defined by ITU-R recommendations.

These studies showed that the long-term interference protection criterion is always met. This is due to the density of UA in operation, as defined in ITU-R Report M.2171 [5]. The short-term interference criterion is more difficult to meet due to the very short time duration allowed, equivalently less than 3 seconds per month, so that a single UAS exceedance event will likely exceed the protection criterion.
The initial sharing studies showed that the short-term interference criteria would still be met, but when proposed higher FS antenna gains were analyzed, there were some UAS operational scenarios where the short-term protection criterion was not met. These occurred at high latitudes and lower altitudes and were dependent on the FS antenna gain analyzed.

Constraints can be imposed on UA operational parameters to prevent instances of harmful interference under these conditions, for example restricting UAS operations to minimum altitudes as a function of latitude. An alternate approach to ensure protection of the FS is to impose a pfd limit on the UAS satellite transmitter emission. The pfd limit states the maximum amount of pfd from a UAS that reaches the earth's surface, as a function of the incident angel of arrival at the earth. Possible pfd limits are now being proposed for consideration by the ITU-R Working Party 5B in preparation for WRC-19.

\section{Recent Proposals for pfd Limits}

The most recent proposals for development of a pfd limit for the UAS satellite transmitter employ two different methods [6]. The first method, Method A, employs a statistical analysis of determining whether the protection criteria for an FS station are met through simulation of UAS operating within line of sight of an FS station. Statistic are gathered with non-stop co-channel lineof sight operation during a period of one month, that is, at all times a UAS is operating somewhere within the FS line of sight, with the UA entering and exiting the FS line of sight area at random locations. Several proposed pfd limits are tested to determine which maximum pfd limit enables the protection criteria to be met.

Figure 2 shows several pfd limits that were tested, denoted as UAS Option 1, 2, 3, and 4, as well as a pfd limit previously proposed to ITU-R Working Party 5B [7] and a pfd limit adopted in ITU-R Recommendation M.1643 [8] for a different aircraft satellite transmission application. UAV Option 4 was the pfd found by Method A to meet the protection criteria. 


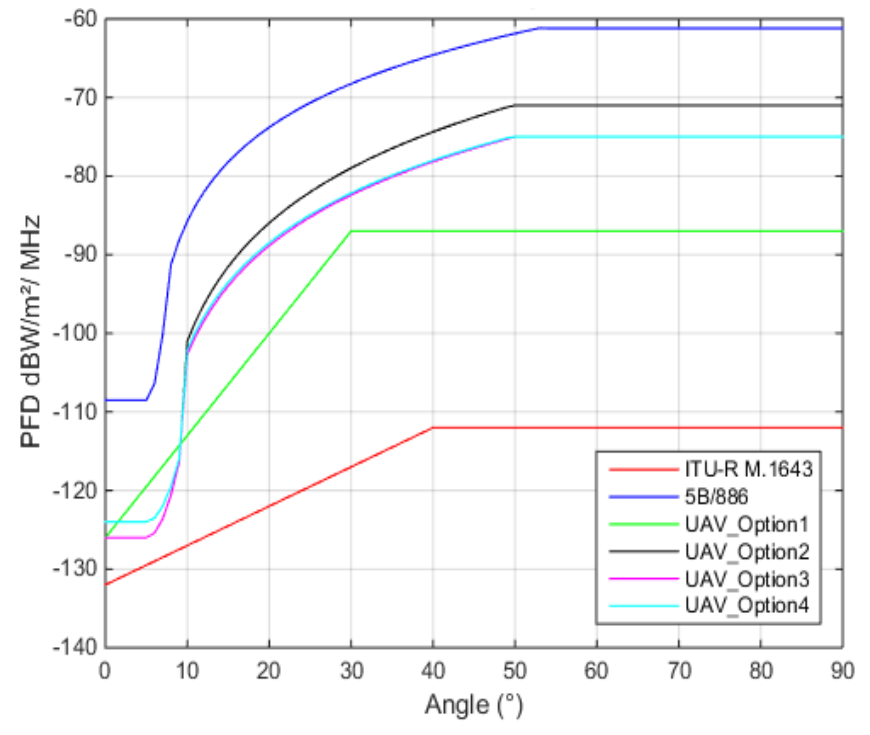

Figure 2 - Possible pfd limits analyzed by Method A, as a function of incident angle to the FS station

It is important to note that Method A assumes a range of FS receiver antenna gains from $-18,28$, 35,45 and $49 \mathrm{~dB}$. The FS antenna gain is a critical parameter of the analysis. Within ITU-R, currently the antenna gains accepted for use in studies are defined in ITU-R Recommendation F.758 [4]. The maximum antenna gain cited by F.758 for $14.4 \mathrm{GHz}$ is $37 \mathrm{~dB}$. The higher values used in Method A represent proposed changes to F.758 that have yet to be adopted.

The second method proposed for deriving the pfd limit, Method B, relies on analysis of the maximum interference criterion and the receiver antenna on-axis and off-axis gain characteristic to derive the required pfd limit. The maximum interference criterion is the short-term criterion of $\mathrm{I} / \mathrm{N}$ at the FS receiver input. The analysis also assumes an FS antenna elevation angle of 5 degrees, which was the maximum required for the analysis and was shown to be the worst-case.

Figure 3 shows the resulting Method B pfd limit, compared to the Method A pfd limit and the pfd limit from ITU-R Recommendation M.1643. The Method B pfd limit is less stringent, particularly at the lower FS incident angles.

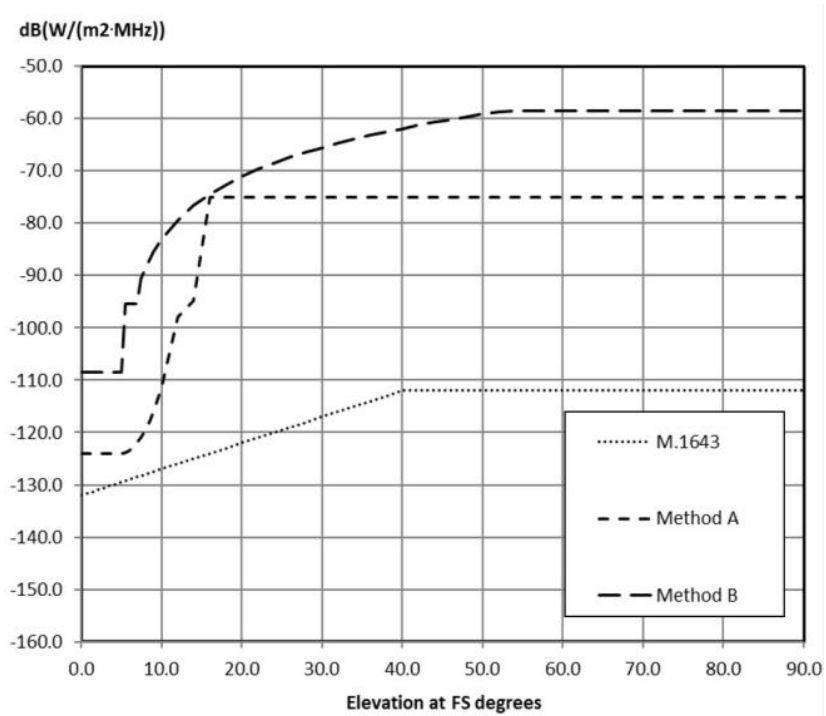

Figure 3 - Comparison of pfd limits proposed by Method $A$ and $B$, as a function of incident angle to the FS station

\section{Possible Refinements of PFD Analyses}

There are several areas where refinement of the pfd analyses is possible. In addition, a more accurate understanding of the propagation characteristics of the interference channel will improve the overall analysis. NASA proposes to conduct measurements and develop channel models for the interference channel, as discussed in the next section.

As noted above, the receive antenna gain of the FS station is a critical element of the analysis. The antenna gains in approved ITU-R recommendations are required to be used for sharing studies. However, Method A described above made use of higher antenna gains on the basis of proposals to change antenna gains contained in ITU-R Recommendation F.758. ITU-R Working Party 5C will decide on revision of F.758 to include additional antenna gains at $14.4 \mathrm{GHz}$.

The density of UAS applied to the analysis of Method A can also be further refined. Method A assumes a UAS always within view of a FS which implies a higher UA density than has been used in previous sharing studies, which relied on projections found in ITU-R Report M.2171. The densities in M.2171 are based on projected peak UA numbers that were developed in order to determine peak spectrum requirements. A more 
accurate analysis would be made by applying the average UAS density. However, the average UAS density has not yet been calculated. A new analysis of UAS average density is therefore needed.

\section{Measuring and modeling the air- ground interference path}

There is no current specific model of the propagation path from an airborne UAS to the ground in the $14.0-14.47 \mathrm{GHz}$ band. NASA is planning to address this deficiency by making propagation path loss measurements of the airground channel and using the resulting data to develop new channel models.

ITU-R Recommendation P.525-2 provides a path loss model for point to point links, shown in Figure 4 [9]. This model is not specific to an airground channel and thus a refinement of this model to accurately model the air-ground channel at 14.0$14.47 \mathrm{GHz}$ is the goal of this activity.

With a point-to-point link it is preferable to calculate the free-space attenuation between isotropic antennas, also known as the free-space basic transmission loss (symbols: $L_{b f}$ or $A_{0}$ ), as follows:

$$
L_{b f}=20 \log \left(\frac{4 \pi d}{\lambda}\right) \quad \mathrm{dB}
$$

where:

$L_{b f}:$ free-space basic transmission loss $(\mathrm{dB})$

$d$ : distance

$\lambda$ : wavelength, and

$d$ and $\lambda$ are expressed in the same unit.

Equation (3) can also be written using the frequency instead of the wavelength.

$$
L_{b f}=32.4+20 \log f+20 \log d \mathrm{~dB}
$$

where:

$f: \quad$ frequency $(\mathrm{MHz})$

$d: \quad$ distance $(\mathrm{km})$.

Figure 4 - Calculation of free space attenuation for a point-to-point link, ITU-R Recommendation P.525-2
The free space path loss equation from P.525-2 has been applied in all previous analyses and simulations. In augmenting this equation with empirical path loss data for the $14.0-14.47 \mathrm{GHz}$ airground channel, the accuracy of interference analyses and development of appropriate pfd limits for the protection of FS receivers can be improved. In addition, cumulative distribution function curves illustrating the amplitude and non-symmetrical characteristics and the relationship to theoretical free-space path loss will be developed, providing new information on the expected interference effects.

The flight test campaign to obtain empirical path loss data is being planned by NASA. The key elements of the test system include a Ku-Band omni-directional antenna mounted on the bottom of the test aircraft, the NASA GRC S3-B, to be used for transmitting the test signal to the ground. To serve as a receiver terminal, a $2 \mathrm{ft}$. dish antenna mounted on a $30 \mathrm{ft}$. portable tower will simulate an FS receive station. The antenna has a $2.4^{0}$ halfpower beamwidth. Mounted on the same tower below the FS antenna will be a horn antenna with a wider beamwidth of $\sim 30^{\circ}$ to provide received signal strength (RSS) data for characterizing the path loss. This method will assure that high amplitude RSS data will continue to be collected even outside of the main beam of the FS antenna. Data collection will be accomplished through a tracking receiver and spectrum analyzer.

Table 2 shows the calculated path loss using P.525-2 from an aircraft at an altitude of 5,000 ft, with the ground distance and corresponding pfd angles. The aircraft will fly down the boresight of the antenna at a constant $5,000 \mathrm{ft}$ altitude in this example. A pfd angle of $90^{\circ}$ corresponds to the aircraft directly over the FS antenna. Actual measured path loss numbers will be entered into the table, averaged over several flights.

Figure 5 shows the relationship between aircraft-ground station range and elevation angle (i.e. incident angle on the ground) for several altitudes. Incident angles between $0^{0}$ and $20^{\circ}$ are of the most interest, since this is where the pfd limits will be the most difficult to meet for UAS transmitters. Incident angles above $20^{\circ}$ will experience the effects of fuselage attenuation, reducing the pfd reaching the earth [6]. 
Table 2 - Example flight test with the aircraft at an altitude of $5000 \mathrm{ft}$.

\begin{tabular}{|c|c|c|c|}
\hline $\begin{array}{c}\text { PFD } \\
\text { Angle }\end{array}$ & $\begin{array}{c}\text { Distance } \\
(\mathbf{k m})\end{array}$ & $\begin{array}{c}\text { TU-R P.525-2 } \\
\text { FSPL (dB) }\end{array}$ & $\begin{array}{c}\text { Actual } \\
\text { Path Loss }\end{array}$ \\
\hline 1.0 & 67.0784 & 152.1424 & TBD \\
\hline 3.0 & 27.9072 & 144.5357 & TBD \\
\hline 5.0 & 17.1513 & 140.3286 & TBD \\
\hline 10.0 & 8.6079 & 134.4404 & TBD \\
\hline 15.0 & 5.6768 & 130.9926 & TBD \\
\hline 20.0 & 4.1823 & 128.5781 & TBD \\
\hline 25.0 & 3.2656 & 126.7432 & TBD \\
\hline 30.0 & 2.6380 & 125.2844 & TBD \\
\hline 40.0 & 1.8155 & 123.1041 & TBD \\
\hline 50.0 & 1.2784 & 121.5811 & TBD \\
\hline 60.0 & 0.8796 & 120.5160 & TBD \\
\hline 70.0 & 0.5545 & 119.8071 & TBD \\
\hline 80.0 & 0.2687 & 119.3999 & TBD \\
\hline 90.0 & 0.0000 & 119.2669 & TBD \\
\hline
\end{tabular}

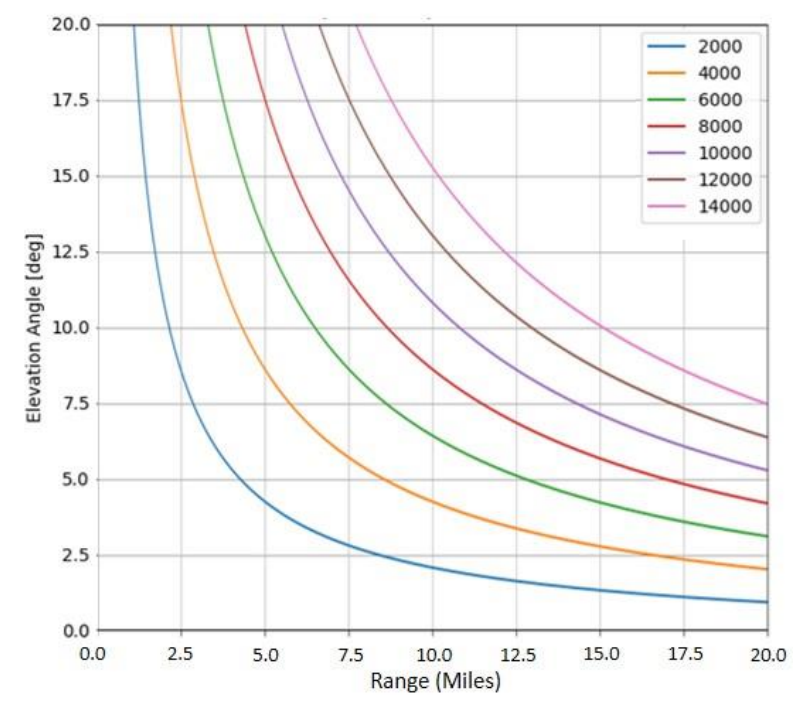

Figure 5 - Range vs. elevation angle for several aircraft altitudes (ft)

Figure 6 shows the geometry of antenna boresight relative to the Earth's surface. The location where the aircraft will be within the main beam of the antenna is denoted by the arc labeled " $L$ " in Figure 6, assuming the FS receive antenna $3 \mathrm{~dB}$ beamwidth of $2.4^{0}$. The FS antenna elevation is $+5^{0}$, at a height of $30 \mathrm{ft}$.

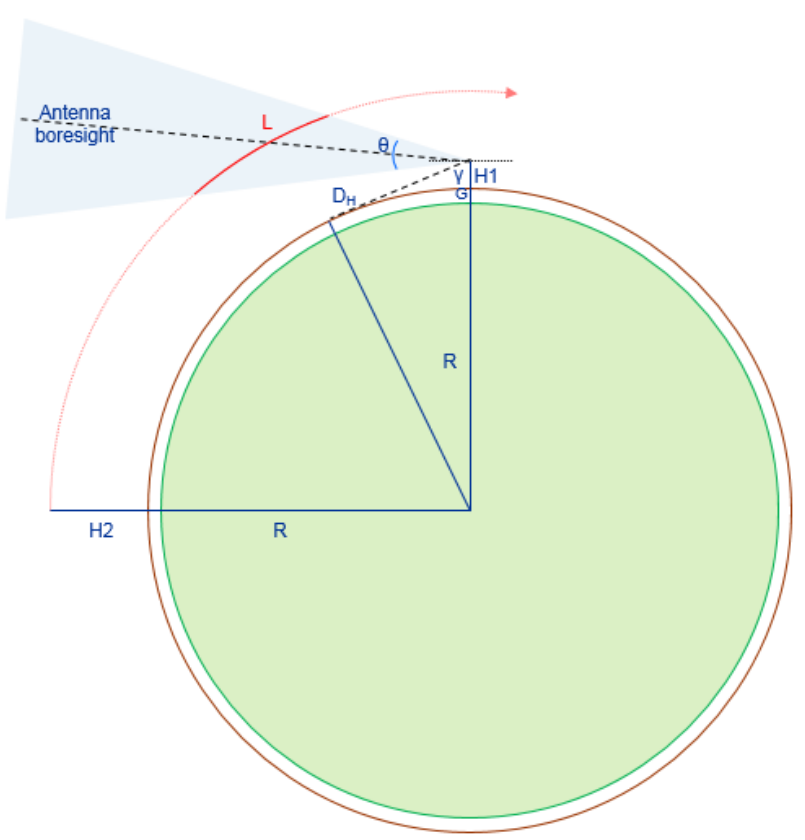

Figure 6 - Geometry of flight tests, showing the location where the aircraft is flying within the main beam of the receive FS antenna.

Several sets of flight tests are planned and will include flight paths directly through the boresight as well as across (orthogonal to) the boresight to effectively plot the path loss across the entire antenna pattern.

\section{Summary}

The ability of UAS to operate in nonsegregated airspace is dependent upon a highly reliable and robust $\mathrm{C} 2$ communications link. Both terrestrial LOS and satellite BLOS links are required. Minimum operational performance standards have been completed for terrestrial LOS C2 links and are in development for satellite BLOS C2 links.

Spectrum allocations have been made at an international level for both LOS and BLOS requirements. WRC-15 adopted Resolution 155 to provide allocations for UAS C2 links under the Fixed Satellite Service in both Ku-Band and KaBand. For the Ku-Band case, the uplink band at 14.0-14.47 GHz shares a co-primary allocation with terrestrial FS systems. Protection of the FS 
receivers from harmful interference caused by UAS satellite transmitters will require a pfd limit to be imposed on UAS transmissions. The pfd limit will be decided at WRC-19.

Two methods have been proposed to identify the appropriate pfd limit on UAS transmissions. The methods produce different results and are based on different assumptions and system parameters. In particular, FS receive antenna gains are being used in the different methods, and the correct gains have yet to be agreed upon. An update of the expected densities of UAS making use of BLOS satellite C2 links, and in particular the average density, would also potentially improve the pfd analyses

Another refinement of pfd analyses can be obtained through the development of specific path loss models for the air-ground channel at 14.014.47 GHz. NASA is in the planning stages for a flight test campaign to perform path loss measurements for the air-ground channel, from which specific path loss models will be developed. Application of these models to the pfd analysis effort may provide better insight into the expected interference levels and result in an improved pfd limit result.

\section{References}

[1] Kerczewski R. J., Wilson, J. D., and Bishop, W. D., "Assessing Spectrum Compatibility for BeyondLine-of-Sight UAS Control and Non-Payload Communications", $2014 \quad$ Integrated Communications, Navigation and Surveillance Conference, April 2014.

[2] Kerczewski R. J., Wilson, J. D., and Bishop, W. D., "Parameter Impact on Sharing studies Between UAS CNPC Satellite Transmitters and Terrestrial Systems," 2015 Integrated Communications, Navigation and Surveillance Conference, April 2015.

[3] Kerczewski R. J., Wilson, J. D., and Bishop, W. D., "UAS CNPC Satellite Link Performance Sharing Spectrum with Terrestrial Systems", 2016 IEEE Aerospace Conference, March 2016.

[4] ITU-R Recommendation F.758-5 "System parameters and considerations in the development of criteria for sharing or compatibility between digital fixed wireless systems in the fixed service and systems in other services and other sources of interference", March 2012.

[5] ITU-R Report M.2171 "Characteristics of unmanned aircraft systems and spectrum requirements to support their safe operation in nonsegregated airspace”, December 2009.

[6] ITU-R Working Party 5B Document 5B/154E, "Proposal on Studies at Working Party 5B for Resolution 155 (WRC-15)", United States of America, October, 2016.

[7] ITU-R Working Party 5B Document 5B/886, "Technical and operational characteristics, interference and regulatory environments associated with the use of frequency bands allocated to the fixed-satellite service not subject to Appendices 30, $30 \mathrm{~A}$ and $30 \mathrm{~B}$ for the control and non-payload communication of unmanned aircraft systems in non-segregated airspace, WRC-15 agenda item 1.5", Germany (Federal Republic of) and United States of America, August 2015.

[8] ITU-R Recommendation M.1643 "Technical and operational requirements for aircraft earth stations of aeronautical mobile-satellite service including those using fixed-satellite service network transponders in the band 14-14.5 GHz (Earth-tospace)", 2003.

[9] ITU-R Recommendation P.525-2, "Calculation of Free Space Attenuation, 1994

\section{Integrated Communications Navigation and Surveillance (ICNS) Conference}

April 18-20, 2017 